\title{
A homozygous missense variant in HSD17B4 identified in a consanguineous Chinese Han family with type II Perrault syndrome
}

Kui Chen ${ }^{\dagger}$, Ke Yang ${ }^{\dagger}$, Su-Shan Luo, Chen Chen, Ying Wang, Yi-Xuan Wang, Da-Ke Li, Yu-Jie Yang, Yi-Lin Tang, Feng-Tao Liu, Jian Wang, Jian-Jun Wu and Yi-Min Sun *i)

\begin{abstract}
Background: Perrault syndrome is a rare multisystem disorder that manifests with sensorineural hearing loss in both sexes, primary ovarian insufficiency in females and neurological features. The syndrome is heterogeneous both genetically and phenotypically.

Case presentation: We reported a consanguineous family (two affected sisters) with Perrault syndrome. The proband had the characteristics of Perrault syndrome: ovarian dysgenesis, bilateral hearing loss and obvious neurological signs. Target genetic sequencing and triplet repeat primed PCR (TP-PCR) plus capillary electrophoresis was conducted to detect causative mutations in the proband. The detected variant was further confirmed in the proband and tested in other family members by Sanger sequencing. Both the proband and her sister were found homozygous for the novel variant HSD17B4 c.298G > T (p.A100S) with their parents heterozygous. Detected by western blot, the protein expression of HSD17B4 mutant was much lower than that of the wild type in SH-SY5Y cells transfected by HSD17B4 wild type or mutant plasmid, which indicated the pathogenicity of the HSD17B4 mutation.

Conclusions: Our findings supported that HSD17B4 was one of the genes contributing to Perrault syndrome with the likely pathogenic variant c.298G > T (p.A100S). Special manifestations of cerebellar impairment were found in cases caused by HSD17B4 mutations. Besides, attention should be paid to distinguish Perrault syndrome from D-bifunctional protein deficiency and hereditary ataxia.
\end{abstract}

Keywords: Perrault syndrome, HSD17B4, Variant, Neurological features, Ovarian dysgenesis, Sensorineural deafness

\section{Background}

Perrault syndrome (PRLTS, MIM233400), a rare autosomalrecessive disorder influenced by sex, is characterized by sensorineural hearing loss in both male and female patients and ovarian dysgenesis in female patients [1]. A wide variety of additional clinical features including peripheral neuropathy, marfanoid habitus, cerebellar ataxia, intellectual disability and spasticity, most neurologically, have also been reported [2-4]. The clinical heterogeneity has prompted a suggested classification of PRLTS into

\footnotetext{
* Correspondence: ys2504@sina.com

${ }^{\dagger}$ Equal contributors

Department \& Institute of Neurology, Huashan Hospital, Fudan University, 12 Wulumuqi Zhong Road, Shanghai 200040, China
}

type I and type II, without and with neurological symptoms respectively $[2,5]$. However, the determinants for different types remain unknown.

With further evidence, it is likely that the genetic heterogeneity has relationship with the phenotypic heterogeneity. Mutations in four genes including HSD17B4 (MIM601860), HARS2 (MIM600783), CLPP (MIM601119) and LARS2 (MIM604544) have been identified as genetic causes of PRLTS [5-9]. However, the association between genotype and phenotype remains uncertain.

HSD17B4 locates on chromosome 5q23.1, encoding an enzyme of $17 \beta$-hydroxysteroid dehydrogenase type 4 [10]. This multifunctional peroxisomal enzyme, also known as D-bifunctional protein (DBP), acts an important role in 
fatty acid $\beta$-oxidation metabolism [11]. Therefore, besides PRLTS, HSD17B4 is also related to DBP deficiency (DBPD, MIM261515). DBPD is an autosomal-recessive and infantile-onset disorder characterized by severe neurological symptoms and accumulation of very long chain fatty acid (VLCFA) in plasma [11, 12]. Compared with DBPD patients, PRLTS patients present with both of hearing loss and gonadal dysgenesis, while their VLCFA level is normal.

Since PRLTS is a rare disease, there are only approximately 40 PRLTS families reported worldwide [13], among which three cases were possibly caused by mutations in $H S D 17 B 4[5,9,14,15]$.

Here we gave the report of a PRLTS family in China and found an HSD17B4 mutation to confirm the relationship. Furthermore, we compared PRLTS and DBPD to clarify the diagnosis. In the end, we summarized the PRLTS cases with HSD17B4 mutations.

\section{Case presentation}

\section{Subjects and clinical evaluation}

We collected a PRLTS family with two affected siblings (Fig. 1) from eastern China. The proband (IV2) was subjected to laboratory tests, audiometric examination, thorough neurological examinations, pelvic ultrasonography (iU22 ultrasonography unit, Philips Medical Systems, Bothell, WA) and cranial magnetic resonance imaging (MRI) (3 T MRI scanner Siemens, Erlangen, Germany). The other family members were evaluated by interviews. The CARE guidelines were followed in this case.

\section{Genetic testing and variants screening}

Genomic DNA samples were extracted from peripheral blood leucocytes of all family members. For the proband (IV2), a panel containing 218 genes of hereditary spastic paraplegia and ataxia was performed by target sequencing of the exons. Gene list of the panel was in Additional file 1: Table S1. High throughput sequencing was carried out via the HiSeq2500 sequencer (Illumina, San Diego, CA). All variants different from the reference sequence were further screened by allele frequency $<1 \%$ according to 1000 Genomes Project (http://www.internationalgenome.org/data), Inhouse database and ESP6500 (evs.gs.washington.edu/ EVS/). The synonymous variants were excluded. The phenotypes of the screened genes were compared with the clinical manifestations of the proband and the inherited modes were considered to further exclude irrelevant genes. Then the mutations left were tested in other members by Sanger sequencing for family segregation.

The dynamic mutations of Spinocerebellar ataxia (SCA) type 1, 2, 3, 6, 7, 12, 17 and Dentatorubral-pallidoluysian atrophy (DRPLA) were detected by triplet repeat primed PCR (TP-PCR) and capillary electrophoresis.

SIFT (http://sift.jcvi.org/), Polyphen-2 (http://genetics. bwh.harvard.edu/pph2/), and MutationTaster (http://www. mutationtaster.org/) computer simulation software programs were used to predict the potential of the variants to affect function.

\section{Construction of recombinant plasmids}

PCR fragments encoding the mutant or wild-type HSD17B4 gene were amplified using a pair of designed primers (forward nucleotide sequence: CGCAAATGGG CGGTAGGCGTG and reverse nucleotide sequence: TAGAAGGCACAGTCGAGG). The cDNA was then cloned into the pcDNA3.1-3xFlag vector via the Xhol and BamHI restriction sites. Both constructs were confirmed by sequencing.

\section{Cell culture and transfection}

SH-SY5Y cells were used throughout this study. The cells were cultured in DMEM media supplemented with

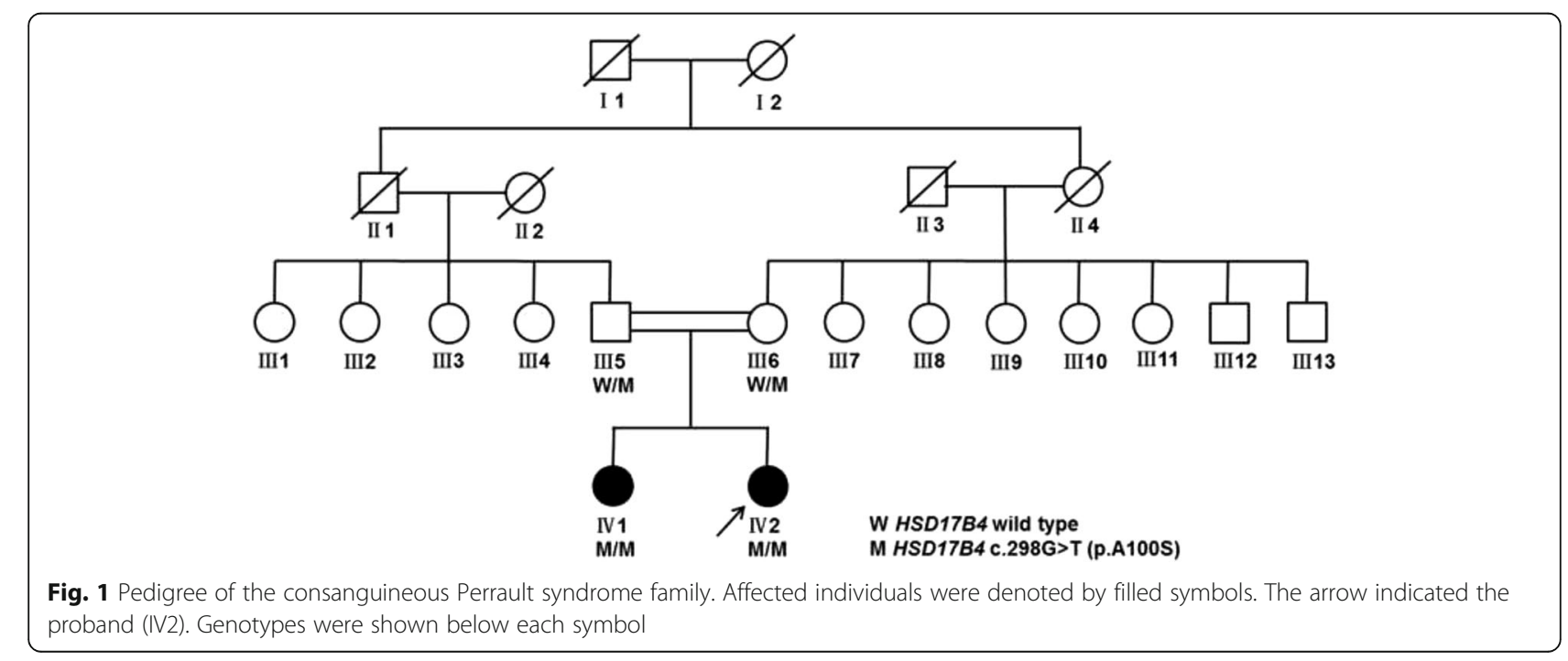


$10 \%$ fetal bovine serum and plated into wells of a sixwell plate with a density of $3 \times 10^{5}$ cells $/ \mathrm{ml}$. Transfections were carried out using Lipofectamine 2000 from Life Technologies (Invitrogen) according to the manufacturer's protocol. The experiments were repeated separatedly for three times.

\section{Western blotting}

Following transfection for $36 \mathrm{~h}$, total proteins were extracted with cell lysis buffer for western blotting (Beyotime) containing of Protease Inhibitor Cocktail (Thermo). Equal amounts of protein were diluted into $5 \times$ loading buffer to $20 \mathrm{ml}$. After electrophoresis, the separated proteins were transferred to Nitrocellulose membranes. Then the membranes were blocked in TBST containing 5\% non-fat milk for $1 \mathrm{~h}$ and incubated overnight with primary antibodies, rabbit anti-HSD17B4 (Dilution 1:100, HPA021479, Sigma) and mouse anti-beta-actin (Dilution 1:1000, \#M20011, Abmart). Next, the membranes were incubated for $1 \mathrm{~h}$ with a matching secondary antibody at room temperature. Protein expression was detected using the Immobilon Western Chemiluminescent HRP Substrate. After normalization to actin, relative protein expression was calculated by Imagelab.

\section{Clinical features}

This family was consanguineous and consisted of the female proband (37-year old), her affected sister (40-year old) and their unaffected relatives (Fig. 1). The proband referred to the neurology clinic initially for ataxia described as the deteriorated ability of balance leading to occasional falls. She also had dysarthria for seven years. In the nervous system examination, she presented with ataxic gait, mild hypertonia in lower limbs, hyperactive deep tendon reflexes, while muscle strength and sensory conduction were normal, and there was no obvious dysmorphology. She performed badly in finger-nose test, alternating movement test and straight-line walking. In addition, she had nystagmus on lateral gaze and hypermetric saccades, but there was no limitation in extraocular movements. We could also find modest dysarthria and mildly intellectual impairment. Her Mini-Mental State Examination score was 25 and considered as mild cognitive impairment according to her education year of 9 [16]. The cranial MRI showed moderate atrophy of the cerebellum (Fig. 2).

The patient also had secondary amenorrhea which needed the endocrine therapy to keep the regular menstrual cycle. But she had stopped the therapy a few years ago. According to her hormone profile, luteinizing hormone $(\mathrm{LH})$ and follicle stimulating hormone (FSH) were elevated, and estradiol was low (Table 1). Furthermore, the uterus appeared small $(40 \times 25 \times 20 \mathrm{~mm})$ with endometrial stripes and indistinct ovaries on ultrasound. These results were consistent with hypergonadotropic hypogonadism.

Another clinical feature was hearing impairment. The proband had hearing dysfunction from childhood without great progression in these years. Audiometric examination showed a bilaterally sensorineural hearing deficit for higher frequencies (Fig. 3).

In addition, her VLCFA was normal (Table 2).

The proband's sister (IV1) had similar symptoms as the proband. She had primary amenorrhea and tried several therapies all of which ended with failure. She also suffered from the hearing loss. And she had the tendency to have a fall in daily life. She was unwilling to take the hormone test, ultrasound test of uterus, VLCFA or cerebral MRI scan. Both of their parents were healthy.

\section{Genetic information}

The mean depth of target sequencing of the patient was $1083.5 \times$ and the coverage was $100 \%$. The percentage of the target region with mean depth $>20 \times$ was $100 \% .29$ variants left after screening by the variants frequencies

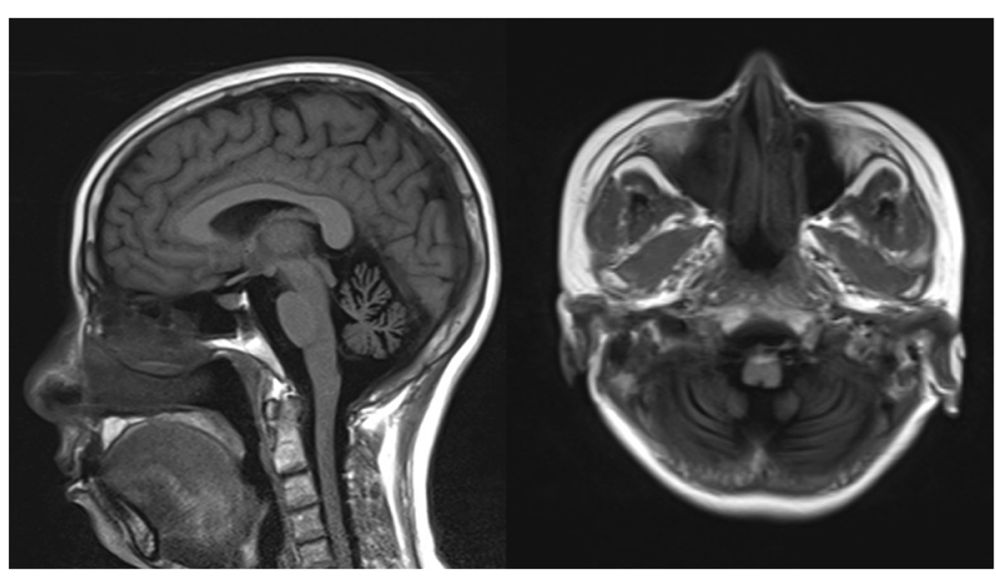

Fig. 2 Brain MRI images for the proband (IV2). Marked and diffuse cerebellar atrophy was present in the proband 
Chen et al. BMC Medical Genetics (2017) 18:91

Page 4 of 9

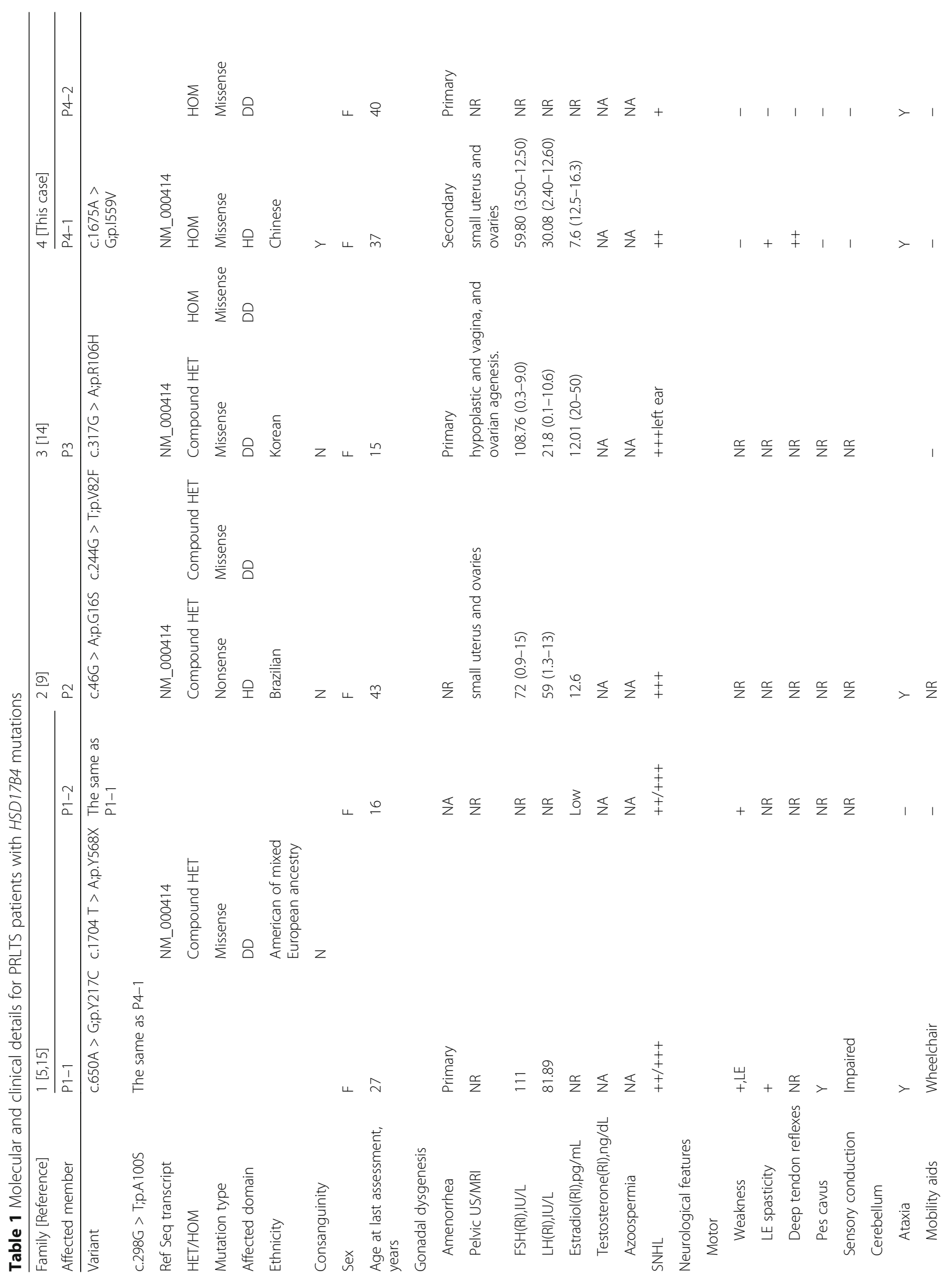




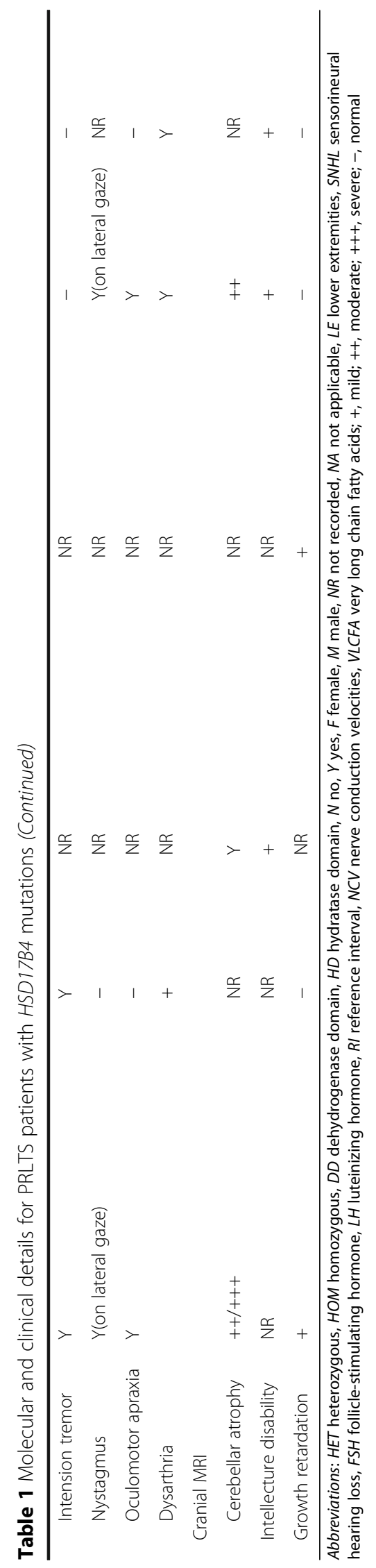



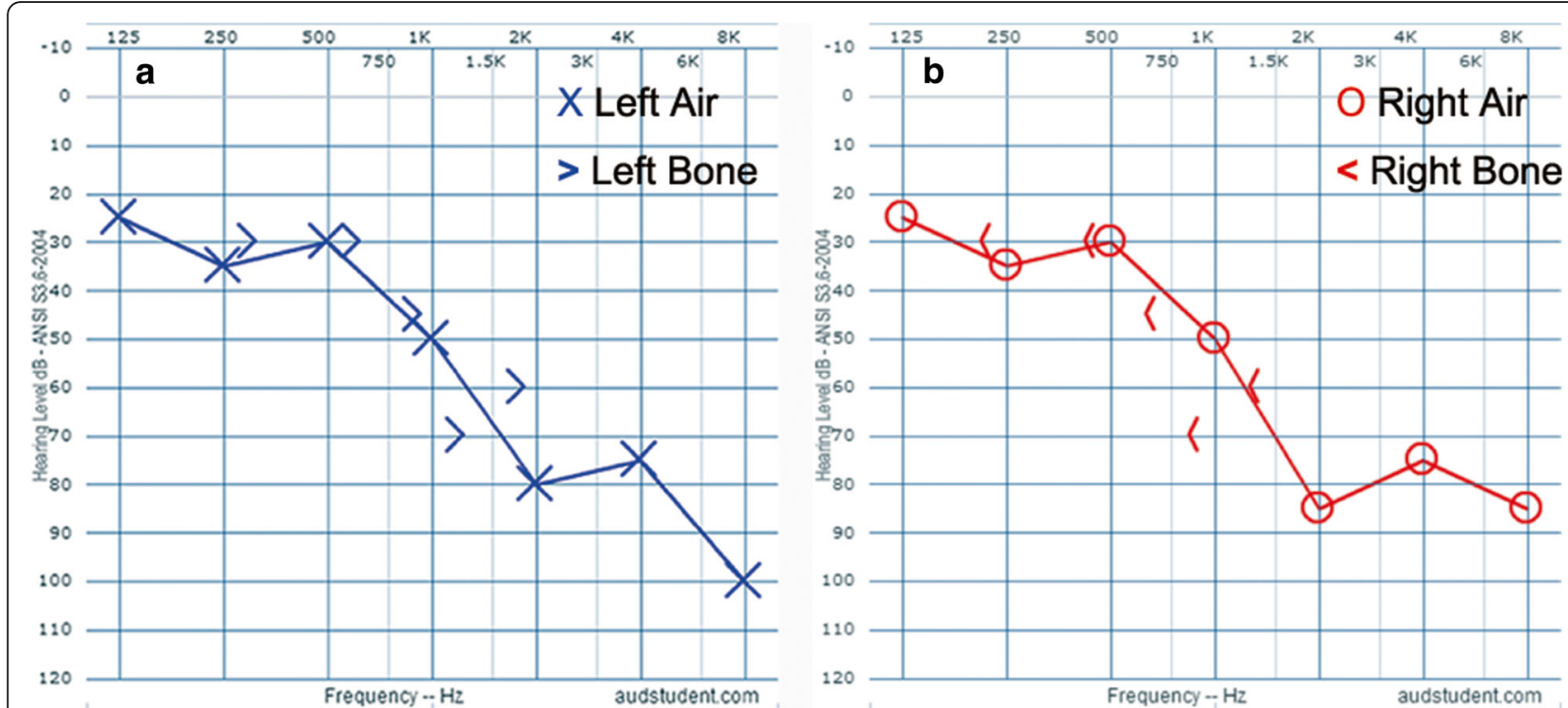

Fig. 3 Audiograms from the proband at the age of 37. a Audiogram of the left ear. $\mathbf{b}$ Audiogram of the right ear. In pure-tone audiometry measurements, similar air and bone thresholds are indicative of hearing loss due to dysfunction of the inner ear, which demonstrates bilaterally sensorineural hearing loss especially at high frequency. Audiograms were created using the AudGen online tool (version 0.71) (http://audsim.com/audgen/s

and synonymous status (Additional file 2: Table S2). According to the autosomal recessive inheritance mode in this family, we complemented the variants by homozygosity mapping. Two variants of c.298G > T (p.A100S) (accession no. NM_001199292) in HSD17B4 and c.188A > G (p.D63G) in KCNC3 (NM_004977) were found with a sequencing depth of $663 \times$ and $66 \times$ respectively. The variant in $K C N C 3$ was excluded since it was associated with spinocerebellar ataxia 13 whose manifestations were different from this patient. This variant of p.A100S in HSD17B4 was not found in 1000 Genomes Project, Inhouse or ESP6500 database. The predicted values of this variant were 0,1 and 1 in SIFT, PolyPhen and MutationTaster respectively, which resulted in the conclusion of a damaging variant in silico algorithms. Sanger sequencing of other genes associated with PRLTS, including HARS2, CLPP and LARS2, was conducted with negative results. Primer information could be found in Additional file 3: Table S3.

Table 2 VLCFA concentration of the proband of the Perrault syndrome pedigree

\begin{tabular}{llll}
\hline VLCFA & Units & The proband (IV2) & Reference range \\
\hline C22:0 & $\mathrm{nmol} / \mathrm{ml}$ & 47.6 & $\leq 96.3$ \\
$\mathrm{C} 24: 0$ & $\mathrm{nmol} / \mathrm{ml}$ & 22.1 & $\leq 91.4$ \\
$\mathrm{C} 26: 0$ & $\mathrm{nmol} / \mathrm{ml}$ & $<0.40$ & $\leq 1.30$ \\
$\mathrm{C} 24: \mathrm{C} 22$ & & 0.47 & $\leq 1.39$ \\
$\mathrm{C} 26: \mathrm{C} 22$ & & $<0.008$ & $\leq 0.023$
\end{tabular}

Abbreviations: VLCFA very long chain fatty acids
The variant was then confirmed in the proband and investigated in other family members by Sanger sequencing. Her sister was also found homozygous, with both parents heterozygotes (Figs. 1 and 4).

The dynamic mutations in SCA1-3, 6, 7, 12, 17 and DRPLA were not found.

\section{Expression of the mutant gene}

Western blot analysis revealed that the $79 \mathrm{kD}$ full-length DBP protein and the $35 \mathrm{kD}$ dehydrogenase domain were detected in cells transfected with a wild-type construct. However, there was remarkable reduction in protein expression resulted from the variants c.298G > T (p.A100S) in $H S D 17 B 4$. There was significant difference between the protein expression levels of the wild type and the variant (Fig. 5).

\section{Discussion and conclusions}

The proband in this family had clinical hallmarks of Perrault syndrome. Given that she had gonadal dysgenesis, hearing loss and neurological disorders, she could be categorized as type II Perrault Syndrome. In this family, we identified a novel missense variant HSD17B4 p.A100S. According to the guidelines of interpretation of sequence variants of the American College of Medical Genetics and Genomics (ACMG), this mutation was classified as a likely pathogenic variant [17]. The patient's familial history and clinical characteristics were specific. And variants in other suspected genes were not found by the target sequencing. Moreover, mutations in 

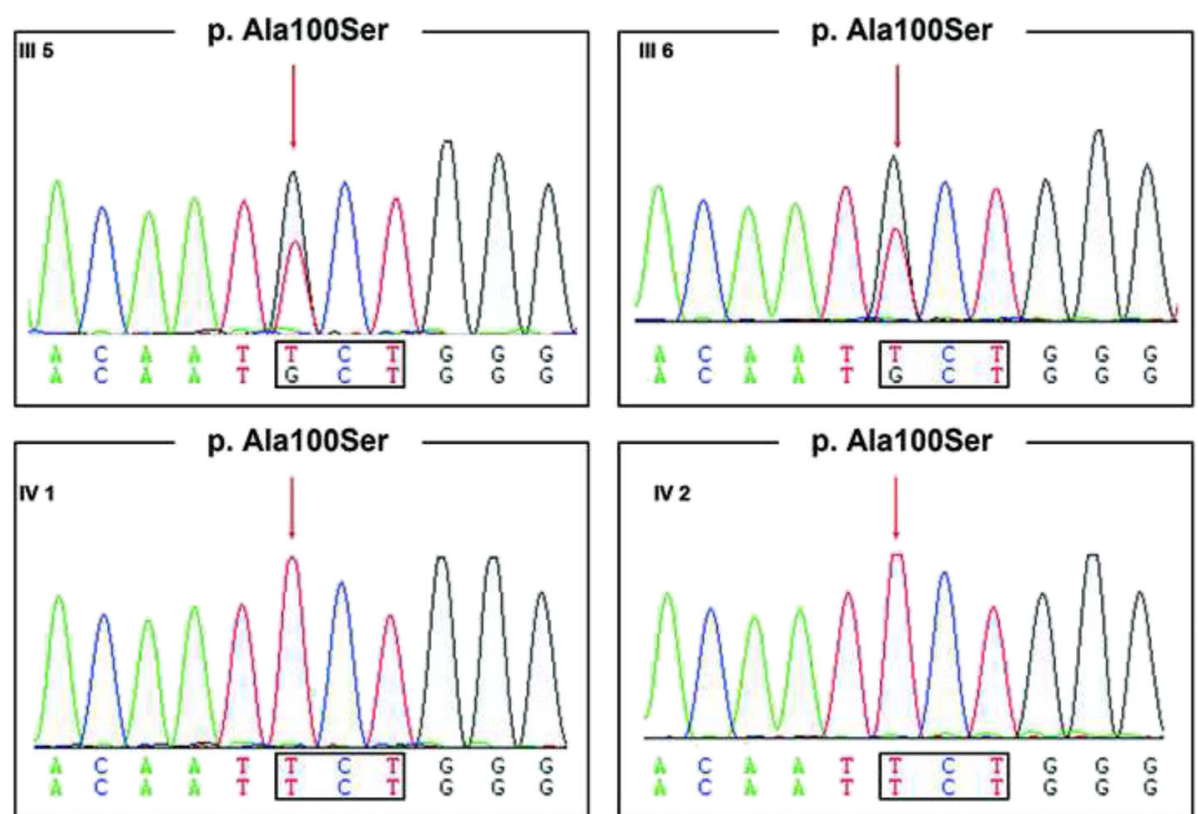

Fig. 4 Sanger sequences of the mutation in HSD17B4 in the affected sisters and their parents. The position of the G > T transition, which led to alanine to serine substitution, was indicated by an arrow. The mutation could be found in heterozygous parents (III5 and III6) and affected patients (IV1 and IV2)

HSD17B4 would result in the alteration in the dehydrogenase domain of DBP and had been identified as the possible cause for Perrault syndrome in several families $[14,18]$. In this case we have demonstrated that HSD17B4 p.A100S had a damaging effect on the gene product by western blot. On the basis of these evidences, this homozygous variant was considered as the probable cause of the disease.

As mentioned above, HSD17B4 encodes the protein peroxisomal enzyme DBP that catalyzes the $\beta$-oxidation of VLCFA. There are three domains in DBP: a 3hydroxyacyl-CoA dehydrogenase domain, a 2-enoyl-CoA

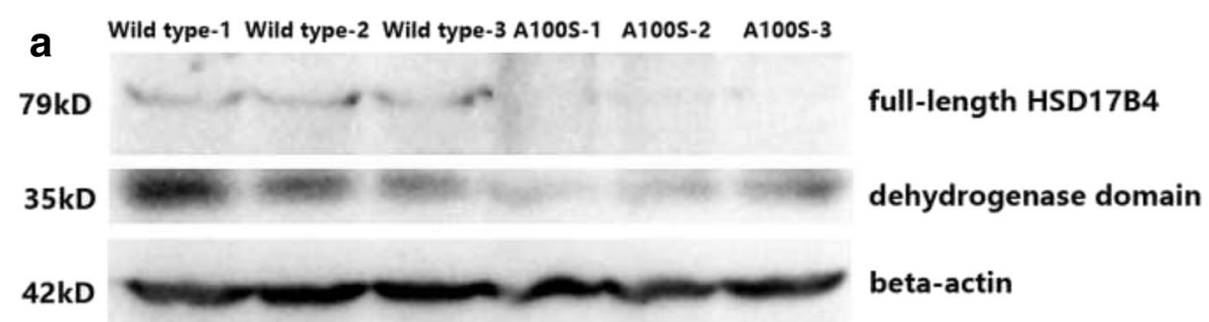

b

HSD17B4-79kD : $\beta$-actin

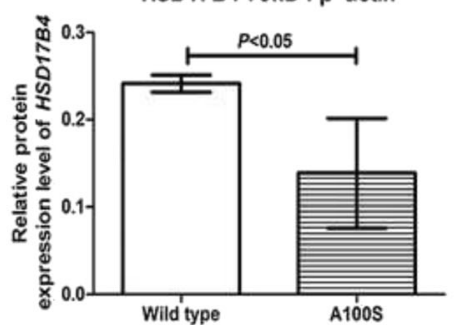

HSD17B4-35kD : $\beta$-actin

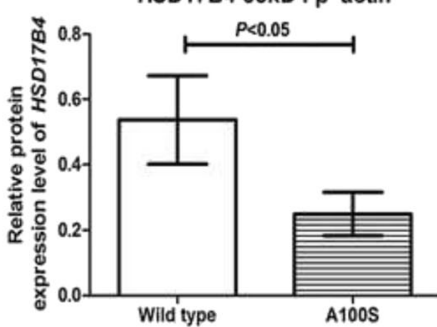

Fig. 5 Analysis of wild-type and mutant HSD17B4 protein expression by Western blot. a Compared by T-test, expression of the variant c.298G > T (p.A100S) was lower than expression of the wild-type. $\mathbf{b}$ There was significant difference between protein expressions for transfected wild-type and mutant plasmids 
hydratase domain, and a sterol carrier protein 2-like domain [19]. After peroxisomal import, a hydratase unit is obtained from the proteolytically cleaving of DBP, with a dehydrogenase unit produced meanwhile $[20,21]$. These two domains catalyze the hydration and dehydrogenation steps in $\beta$-oxidation of the VLCFA sequentially [22]. The mutant p.A100 residue in this case was located at the dehydrogenase region of DBP [5]. HSD17B4 p.A100S was predicted by several algorithms to be damaging.

HSD17B4 variants can lead to PRLTS as well as DBPD. These two diseases also have some similar manifestations so that it is necessary to differentiate PRLTS from DBPD. DBP dysfunction could result in the accumulation of $\beta$-oxidation substrates such as VLCFA, which is one of the most significant characteristics of DBPD typesI, II and III. In most cases of DBPD typesI, II and III, the patients presented with severe manifestations including hypotonia and seizures by one month of age and die within the first two years of life [12]. These patients also had dysmorphic features, visual and hearing impairment, and growth retardation [11]. According to the deficient activity, DBPD can be divided into three subtypes [23]. Type I is the result of deficiencies of both the hydratase and dehydrogenase subunits of DBP, while types II and III have isolated deficiency of hydratase or dehydrogenase. However, a novel type of DBPD has been proposed recently [24]. Patients with the juvenile-onset type, also known as type IV, show mildly clinical features of hearing loss, ataxia, retinal atrophy and fertility in males and females with compound heterozygous mutations affecting both subunits in separate alleles. The juvenile-onset DBPD seems to have more various symptoms and higher VLCFA level than PRLTS. However, the VLCFA in some DBPD patients could be normal as well $[2,25]$. On account of the similar cause and manifestation, PRLTS due to HSD17B4 mutations and juvenile-onset DBP deficiency may be the same disease or part of the same disease spectrum. In our case, besides milder course of the disease, hearing loss and neurological signs, the proband had moderate ovarian dysgenesis, an essential characteristic of PRLTS. In addition, her VLCFA level was normal, and there was only cerebellar atrophy in MRI which was different from the "peroxisomal pattern" [26]. The "peroxisomal pattern" was considered as a diagnostic clue for DBPD when the VLCFA level was normal. Typically, there was cerebral and cerebellar leukencephalopathy, perisylvic polymicrogyria, and frontoparietal pachygyria in the "peroxisomal pattern". While in the cases of PRLTS, there was usually cerebellar atrophy. These features made us tend to the diagnosis of PRLTS rather than the juvenile-onset DBPD. Nevertheless it remained to investigate the relationship between these two diseases associated with $H S D 17 B 4$.
Based on this case, we summarized definite or probable PRLTS cases involving HSD17B4 mutations (Table 1) $[5,9,14,15]$. Interestingly, these patients presented with various neurological symptoms, including signs of cerebellar dysfunction, ocular anomalies and intellectual disability. Among these, cerebellar manifestations were obvious, which could also be demonstrated by the cerebellar atrophy in MRI. Compared with that, the symptoms relevant to peripheral nervous system seemed to be milder. Hearing loss and gonadal dysgenesis, the typical features of PRLTS, were developed in all patients with HSD17B4 mutations. In other PRLTS cases without HSD17B4 variants, either marfanoid features or short stature was reported $[27,28]$, both of which were not shown characteristically in the patients with $H S D 17 B 4$ mutations except for pes cavusin. In our case, ataxia was the chief complaint and this might lead to misdiagnosis if the symptoms of ovarian dysgenesis and hearing loss were ignored.

This report genetically confirmed a PRLTS family in China. This family was consanguineous and the patients were homozygous for HSD17B4 c.298G > T (p.A100S). In review of PRLTS patients with HSD17B4 mutation, we found that these patients presented with various neurological symptoms. More cases could be collected to find whether there was an association between HSD17B4 mutations and PRLTS phenotype. Besides, the mechanism remains to investigate that how the mutations in HSD17B4 lead to PRLTS and DBPD.

\section{Additional files}

Additional file 1: Table S1. Gene list in the panel performed by target sequencing of the exons. (DOCX $29 \mathrm{~kb}$ )

Additional file 2: Table S2. List of Variants in the proband. 29 variants were left after screening by the variants frequencies and synonymous status. (XLSX $26 \mathrm{~kb}$ )

Additional file 3: Table S3. Primer information of HARS2, CLPP and LARS2 for Sanger sequencing. (XLS 27 kb)

\section{Abbrevations \\ ACMG: American College of Medical Genetics and Genomics; DBP: D-bifunctional protein; DBPD: DBP deficiency; DRPLA: Dentatorubral-pallidoluysian atrophy; FSH: Follicle stimulating hormone; LH: Luteinizing hormone; OMIM: Online Mendelian Inheritance in Man; PRLTS: Perrault syndrome; SCA: Spinocerebellar ataxia; VLCFA: Very long chain fatty acid; VUS: Variant of uncertain significance}

\section{Acknowledgements}

We sincerely appreciate the patients and their relatives for their help and willingness in this study.

\section{Funding}

This work was supported by grants from the National Natural Science Foundation of China (No. 81571232,No.81371413, No. 81401048), the project of Shanghai Municipal Commission of Health (XBR2013088, 20154Y0004) and the project from the Science and Technology Commission of Shanghai Municipality (15ZR1435800).

\section{Availability of data and materials}

Target genetic sequencing raw data are available from the corresponding author on reasonable request. 


\section{Authors' contributions}

Participation in analyzing and interpreting the sequencing data: KC, KY, SSL and YMS. Participation in preparing the manuscript: KC, CC and YW. Participation in gathering the clinical data for the study: YXW, DKL, YJY and YLT. Review the pertinent raw data on which the results and conclusions of this study are based: YMS, JJW, FTL and JW. All authors read and approved the final manuscript.

\section{Ethics approval and consent to participate}

The study was approved by the ethics committee of Huashan Hospital. Written informed consents were obtained from all participants prior to their inclusion in the study.

\section{Consent for publication}

Consent to publish pictures and clinical data was obtained.

\section{Competing interests}

The authors declare that they have no competing interests.

\section{Publisher's Note}

Springer Nature remains neutral with regard to jurisdictional claims in published maps and institutional affiliations.

\section{Received: 15 November 2016 Accepted: 14 August 2017} Published online: 23 August 2017

\section{References}

1. Perrault M, Klotz B, Housset E. Two cases of turner syndrome with deafmutism in two sisters. Bull Mem Soc Med Hop Paris. 1951;67(3-4):79-84.

2. Fiumara A, Sorge G, Toscano A, Parano E, Pavone L, Opitz JM. Perrault syndrome: evidence for progressive nervous system involvement. Am J Med Genet A. 2004;128A(3):246-9.

3. Linssen WH, Van den Bent MJ, Brunner HG, Poels PJ. Deafness, sensory neuropathy, and ovarian dysgenesis: a new syndrome or a broader spectrum of Perrault syndrome? Am J Med Genet A. 1994;51(1):81-2.

4. Gottschalk ME, Coker SB, Fox LA. Neurologic anomalies of Perrault syndrome. Am J Med Genet. 1996;65(4):274-6.

5. Pierce SB, Walsh T, Chisholm KM, Lee MK, Thornton AM, Fiumara A, Opitz JM Levy-Lahad E, Klevit RE, King MC. Mutations in the DBP-deficiency protein HSD17B4 cause ovarian dysgenesis, hearing loss, and ataxia of Perrault syndrome. Am J Med Genet. 2010:87(2):282-8.

6. Pierce SB, Chisholm KM, Lynch ED, Lee MK, Walsh T, Opitz JM, Li W, Klevit RE, King MC. Mutations in mitochondrial histidyl tRNA synthetase HARS2 cause ovarian dysgenesis and sensorineural hearing loss of Perrault syndrome. Proc Natl Acad Sci U S A. 2011;108(16):6543-8.

7. Pierce SB, Gersak K, Michaelson-Cohen R, Walsh T, Lee MK, Malach D, Klevit RE, King MC, Levy-Lahad E. Mutations in LARS2, encoding mitochondrial leucyltRNA synthetase, lead to premature ovarian failure and hearing loss in Perrault syndrome. Am J Med Genet. 2013:92(4):614-20.

8. Jenkinson EM, Rehman AU, Walsh T, Clayton-Smith J, Lee K, Morell RJ, Drummond MC, Khan SN, Naeem MA, Rauf B, et al. Perrault syndrome is caused by recessive mutations in CLPP, encoding a mitochondrial ATPdependent chambered protease. Am J Med Genet. 2013;92(4):605-13.

9. Demain LA, Urquhart JE, O'Sullivan J, Williams SG, Bhaskar SS, Jenkinson EM, Lourenco CM, Heiberg A, Pearce SH, Shalev SA, et al. Expanding the Genotypic Spectrum of Perrault syndrome. Clin Genet. 2017;91(2):302-312.

10. Lines MA, Jobling R, Brady L, Marshall CR, Scherer SW, Rodriguez AR, Lee L, Lang $A E$, Mestre TA, Wanders RJ, et al. Peroxisomal D-bifunctional protein deficiency: three adults diagnosed by whole-exome sequencing. Neurology. 2014;82(11):963-8.

11. de Launoit $Y$, Adamski J. Unique multifunctional HSD17B4 gene product: 17beta-hydroxysteroid dehydrogenase 4 and D-3-hydroxyacyl-coenzyme a dehydrogenase/hydratase involved in Zellweger syndrome. J Mol Endocrinol. 1999:22(3):227-40

12. Ferdinandusse $S$, Denis S, Mooyer PA, Dekker C, Duran M, Soorani-Lunsing RJ, Boltshauser E, Macaya A, Gartner J, Majoie CB, et al. Clinical and biochemical spectrum of D-bifunctional protein deficiency. Ann Neurol. 2006:59(1):92-104

13. Sampathkumar G, Veerasigamani N. Perrault syndrome - a rare case report. J Clin Diagn Res. 2015;9(3):Od01-2.
14. Kim MJ, Kim SJ, Kim J, Chae H, Kim M, Kim Y. Genotype and phenotype heterogeneity in perrault syndrome. J Pediatr Adolesc Gynecol. 2013;26(1):e25-7.

15. McCarthy DJ, Opitz JM. Perrault syndrome in sisters. Am J Med Genet. 1985;22(3):629-31.

16. Guo QH, Cao XY, Zhou Y, Zhao QH, Ding D, Hong Z. Application study of quick cognitive screening test in identifying mild cognitive impairment. Neurosci Bull. 2010;26(1):47-54.

17. Richards S, Aziz N, Bale S, Bick D, Das S, Gastier-Foster J, Grody WW, Hegde M, Lyon E, Spector E, et al. Standards and guidelines for the interpretation of sequence variants: a joint consensus recommendation of the American College of Medical Genetics and Genomics and the Association for Molecular Pathology. Genet Med. 2015;17(5):405-24

18. Lieber DS, Hershman SG, Slate NG, Calvo SE, Sims KB, Schmahmann JD, Mootha VK. Next generation sequencing with copy number variant detection expands the phenotypic spectrum of HSD17B4-deficiency. BMC Med Genet. 2014;15:30

19. Leenders F, Husen B, Thole HH, Adamski J. The sequence of porcine 80 kDa 17 beta-estradiol dehydrogenase reveals similarities to the short chain alcohol dehydrogenase family, to actin binding motifs and to sterol carrier protein 2. Mol Cell Endocrinol. 1994;104(2):127-31.

20. Jiang LL, Miyazawa S, Souri M, Hashimoto T. Structure of D-3-hydroxyacylCoA dehydratase/D-3-hydroxyacyl-CoA dehydrogenase bifunctional protein. J Biochem. 1997;121(2):364-9.

21. van Grunsven EG, van Berkel E, Mooijer PA, Watkins PA, Moser HW, Suzuki Y Jiang LL, Hashimoto T, Hoefler G, Adamski J, et al. Peroxisomal bifunctional protein deficiency revisited: resolution of its true enzymatic and molecular basis. Am J Med Genet. 1999;64(1):99-107.

22. Baes M, Huyghe S, Carmeliet P, Declercq PE, Collen D, Mannaerts GP, Van Veldhoven PP. Inactivation of the peroxisomal multifunctional protein-2 in mice impedes the degradation of not only 2-methyl-branched fatty acids and bile acid intermediates but also of very long chain fatty acids. J Biol Chem. 2000;275(21):16329-36

23. Moller G, van Grunsven EG, Wanders RJ, Adamski J. Molecular basis of Dbifunctional protein deficiency. Mol Cell Endocrinol. 2001;171(1-2):61-70.

24. McMillan HJ, Worthylake T, Schwartzentruber J, Gottlieb CC, Lawrence SE, Mackenzie A, Beaulieu CL, Mooyer PA, Wanders RJ, Majewski J, et al. Specific combination of compound heterozygous mutations in 17betahydroxysteroid dehydrogenase type 4 (HSD17B4) defines a new subtype of D-bifunctional protein deficiency. Orphanet J Rare Dis. 2012;7:90.

25. Amor DJ, Marsh AP, Storey E, Tankard R, Gillies G, Delatycki MB, Pope K, Bromhead C, Leventer RJ, Bahlo $M$, et al. Heterozygous mutations in HSD17B4 cause juvenile peroxisomal D-bifunctional protein deficiency. Neurology Genetics. 2016;2(6):e114

26. Gronborg S, Kratzner R, Spiegler J, Ferdinandusse S, Wanders RJ, Waterham HR, Gartner J. Typical cMRI pattern as diagnostic clue for D-bifunctional protein deficiency without apparent biochemical abnormalities in plasma. Am J Med Genet A. 2010;152a(11):2845-9.

27. Nikolaou DS, Winston RM. Sporadic Perrault syndrome. J Obstet Gynaecol. 1999;19(4):436-7.

28. Nishi YHK, Kajiyama M, Kawamura I. The Perrault syndrome: clinical report and review. Am J Med Genet. 1988:31(3):623-9.

\section{Submit your next manuscript to BioMed Central and we will help you at every step:}

- We accept pre-submission inquiries

- Our selector tool helps you to find the most relevant journal

- We provide round the clock customer support

- Convenient online submission

- Thorough peer review

- Inclusion in PubMed and all major indexing services

- Maximum visibility for your research

Submit your manuscript at www.biomedcentral.com/submit
Biomed Central 